\title{
Why Sex and Recombination?
}

\author{
N.H. BARTON \\ Institute of Science and Technology, A-3400 Klosterneuburg, Austria \\ Correspondence: nick.barton@ist-austria.ac.at
}

\begin{abstract}
Sex and recombination have long been seen as adaptations that facilitate natural selection by generating favorable variations. If recombination is to aid selection, there must be negative linkage disequilibria - favorable alleles must be found together less often than expected by chance. These negative linkage disequilibria can be generated directly by selection, but this must involve negative epistasis of just the right strength, which is not expected, from either experiment or theory. Random drift provides a more general source of negative associations: Favorable mutations almost always arise on different genomes, and negative associations tend to persist, precisely because they shield variation from selection.

We can understand how recombination aids adaptation by determining the maximum possible rate of adaptation. With unlinked loci, this rate increases only logarithmically with the influx of favorable mutations. With a linear genome, a scaling argument shows that in a large population, the rate of adaptive substitution depends only on the expected rate in the absence of interference, divided by the total rate of recombination. A two-locus approximation predicts an upper bound on the rate of substitution, proportional to recombination rate.

If associations between linked loci do impede adaptation, there can be substantial selection for modifiers that increase recombination. Whether this can account for the maintenance of high rates of sex and recombination depends on the extent of selection. It is clear that the rate of species-wide substitutions is typically far too low to generate appreciable selection for recombination. However, local sweeps within a subdivided population may be effective.
\end{abstract}

Why are sex and recombination so widespread? In bacteria and archaea, it is arguable that recombination is an incidental by-product of other processes. However, the biology of eukaryotes is dominated by sexual reproduction. Meiosis, with its elaborate molecular machinery, is a key characteristic of eukaryotes, and sex has all kinds of consequences: differentiation of male and female sex chromosomes, the physiological mechanisms for producing male and female gametes, everything involved in finding and attracting mates, and so on. Much of Darwin's work was concerned with sex and its consequences - sexual selection, studies of inbreeding, cross-pollination in plants, etc. (see, e.g., Darwin 1871, 1876).

So, why sex and recombination? We have known the answer to this question for a long time: sexual reproduction is an adaptation that facilitates natural selection by bringing together new, favorable combinations of genes. To use a modern term, recombination is an adaptation for "evolvability," for generating variation useful to selection (Wagner and Altenberg 1996; Kirschner and Gerhart 1998; Sniegowski and Murphy 2006). August Weismann (1889), one of the few champions of natural selection in the late 19th century, put this quite clearly:

[T] he communication of fresh [genes] to the germplasm implies an augmentation of the variational tendencies, and thus an increase of the power of adaptation.

The necessity of sexual reproduction for successful selection is shown in nature by the degeneration of non-recombining sex chromosomes (Charlesworth et al.; Bellott and Page, both this volume) and by the relatively short life of asexual species (Maynard Smith 1978; Butlin 2002). The importance of outcrossing has been clear to plant and animal breeders long before it was understood genetically. Arnold (this volume) described the selection in vitro of novel enzyme activities, which could not have been designed from first principles. A less familiar example comes from evolutionary computation, where programs compete against one another, so that selection can generate better algorithms. For example, Koza et al. (2002) used selection to design efficient low-pass filters and found several designs ingenious enough to have previously been patented. There is a great deal of activity in "evolutionary computation," with a wide variety of approaches, but almost all depend on some form of recombination (Mitchell 1998). In all these examples, recombination and selection together are more effective than intelligent design.

It has been surprisingly difficult to show exactly why selection requires recombination to be effective: We still do not have a compelling general explanation for why so many organisms go to so much trouble to mix their genomes with others. The intuitive idea, that sex is good for the species, was accepted until the 1970s, when it was realized that there has to be an advantage to individual genes that increase outcrossing and recombination and, moreover, that this advantage has to be strong enough to outweigh all of the obvious costs (Williams 1975; Maynard Smith 1978). For the past 35 years, much effort has focused on this question, and there has been corresponding progress in clearing the theoretical fog. I argue that although the basic theoretical framework is clear, we still do not know whether selection is generally strong enough, and has the right form, to give a general advantage to sex and recombination. 


\section{RECOMBINATION INCREASES ADDITIVE GENETIC VARIANCE IF THERE ARE NEGATIVE LINKAGE DISEQUILIBRIA}

We start with Fisher's (1930) "Fundamental Theorem of Natural Selection": the increase in mean fitness due to selection on allele frequencies equals the additive genetic variance in fitness (for explanations of Fisher's theorem, see Price [1972] and Edwards [2002]). So, if recombination is to speed up adaptation, it must increase the additive variance in fitness. Moreover, if a modifier allele increases recombination, it will to some extent be associated with the favorable variation that it generates, and will itself increase. So, in principle, an increase in additive genetic variance gives an advantage to both the group and the individual.

Crucially, recombination does not necessarily either increase or decrease the variance in fitness. If genes are already randomly associated in the population, shuffling them will make no difference. Recombination can only have an effect if there are nonrandom associations between genes (i.e., if there is "linkage disequilibrium"). Moreover, if there are positive associations between favorable genes, recombination will reduce the variance in fitness, and so slow down adaptation. For recombination to gain an advantage by increasing the variance in fitness, there must be negative associations: + with -, and with + . Understanding the advantage of recombination comes down to understanding why associations should be predominantly negative - why they should perversely tend to impede selection.

Negative associations can be produced systematically and deterministically by selection, i.e., by negative epistasis. For example, stabilizing selection against extremes of a quantitative trait tends to reduce its variance and generates negative linkage disequilibrium; this slows the response to directional selection (Charlesworth 1993; Burger 1999; Waxman and Peck 1999). The effect of epistasis can be understood, quite generally, from the effect of recombination on the distribution of log fitness (Fig. 1; Barton 1995a). In each generation, selection will increase the mean by precisely the total genotypic variance: If epistasis is generally negative, selection will also reduce the variance. Recombination then has two effects (Fig. 1). First, it will reduce the mean log fitness by breaking up combinations of genes that have just been built up by selection. (If such associations were completely dissipated, the net increase in the mean would equal the additive genetic variance in fitness, which is smaller than the total genotypic variance.) This immediate recombination load will be seen whether epistasis is negative or positive. If epistasis is negative, recombination will also increase the variance, which will give an advantage in the future by increasing the response to selection. This is a very general result that does not depend on exactly how selection acts or on how many genes are involved. Moreover, it involves quantities that in principle can be measured (Charlesworth and Barton 1996; Burt 2000).

Unfortunately, this deterministic explanation seems unlikely. First, it requires that epistasis be negative, but not too negative, and that it should not vary too much

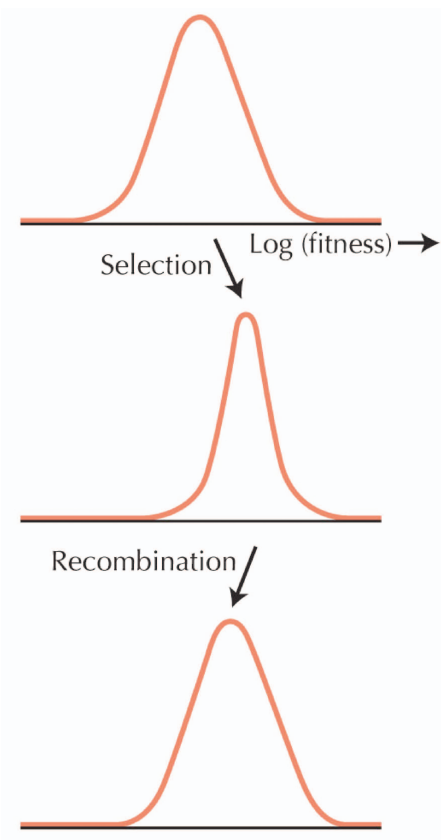

Figure 1. Selection increases the mean log fitness by an amount equal to the total genotypic variance in fitness. If selection favors negative linkage disequilibria, it will also reduce the variance in $\log$ fitness. Recombination causes an immediate reduction in mean fitness by breaking up favorable gene combinations, but it may help future adaptation by breaking up negative associations, thus increasing the variance in log fitness.

between genotypes (Otto and Feldman 1997; Kouyos et al. 2006). Otherwise, the immediate recombination load, due to the breakup of well-adapted combinations, would be too high. Second, there is no evidence that epistasis is usually negative (see, e.g., Elena and Lenski 1997, 2001; Bonhoeffer et al. 2004; de Visser and Elena 2007; Kouyos et al. 2007) and no theoretical reason to expect it to be (but see Szathmary 1993; de Visser and Elena 2007).

\section{DRIFT, RECOMBINATION, AND SELECTION: THE HILL-ROBERTSON EFFECT}

A more general, and more compelling, explanation is that negative associations are produced by random genetic drift - the random fluctuations in genotype frequencies that are inevitable in finite populations - or when a new mutation increases from a single copy. This process, by which random linkage disequilibria tend to be negative, and so to interfere with selection, is known as the Hill-Robertson effect and can be understood in several ways (Hill and Robertson 1966; Maynard Smith 1978). Random drift generates random associations, but by itself, does not to produce positive or negative associations: by chance, ++ or -- genotypes may do better or worse, but on average, there is no systematic bias. It is the interaction between random drift and directional selection that is important: ++ and -- combinations will be strongly selected, and will be quickly swept out of the population, whereas negative associations $(+-$ and -+$)$ will have intermediate fitness, and so will persist. 
Another way to think about the process is to look at the extreme case of asexual reproduction. R.A. Fisher (1930) and Hermann Muller (1932) both pointed out that favorable alleles that arise at about the same time in such a population will compete with one another; in the absence of recombination, only one of them can be fixed (Fig. 2). So, there is a strong advantage to recombination, which can bring different favorable mutations together in the same individual. Here, the randomness is in the origin of new mutations in a single copy. In an extremely large population, with recurrent mutation, the double mutant would arise, there would be no linkage disequilibrium, and recombination would have no effect.

Fisher and Muller described an extreme case, but even when there is some recombination, favorable alleles still compete with one another. Hill and Robertson (1966) first quantified this process, and they gave us yet another way to understand it. From the point of view of a particular allele, selection at linked loci will be experienced as random drift. If the allele happens to be on a fit background, it will increase, whereas if it happens to be on an unfit background, it will decrease. Moreover, fluctuations are especially powerful, because if they are due to linked loci, they will tend to persist for many generations and will cause a large inflation of random drift. Any kind of selection can contribute to Hill-Robertson interference: Elimination of deleterious mutations (known as "background selection"; Charlesworth et al., this volume), fluc-

\section{A Asexual}

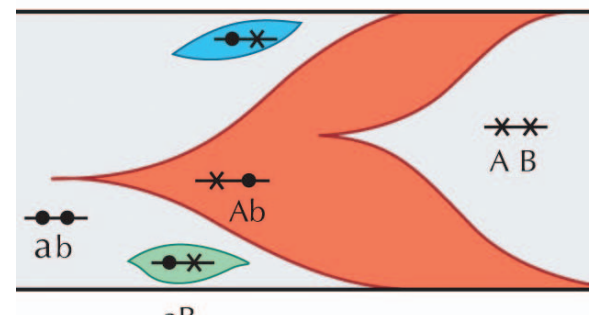

$\mathrm{aB}$

B Sexual

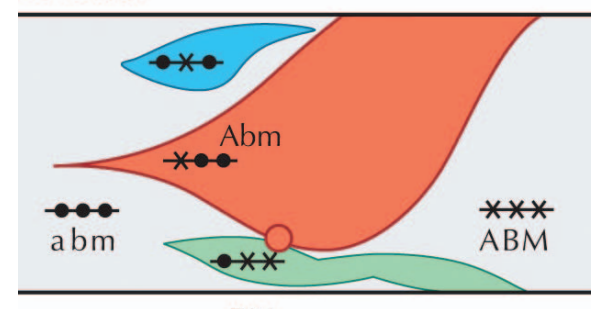

$\mathrm{aBM}$

Figure 2. Favorable mutations must be established sequentially in an asexual population $(A)$. For example, if allele $\mathrm{A}$ is destined to replace a, then any favorable alleles that occur at other loci (B, for instance) can only be fixed if they occur within a genome that carries A. (B) With sexual reproduction, favorable mutations at different loci can be combined; this leads to an advantage to modifiers that causes sex and recombination. A favorable allele $\mathrm{B}$ that occurs with the unfavorable allele a can be fixed if it can recombine into association with A (red circle); if this requires that a modifier allele $\mathrm{M}$ be present, then allele $\mathrm{M}$ will also tend to increase by hitchhiking. tuating selection, or positively selected substitutions will inflate the rate of random drift and so interfere with selection. Several theoretical studies suggest that random drift has a stronger effect on the evolution of recombination than does negative epistasis, even in large populations. Otto and Barton (2001) use simulations of directional selection on standing variation to show that selection for recombination is primarily due to the Hill- Robertson effect, in populations of up to several thousands, even when compared with an optimal level of epistasis. Iles et al. (2003) use similar simulations to show that as the number of loci increases, the effect of drift predominates in ever larger populations. Keightley and Otto (2006) examine selection against deleterious mutations and show that recombination gains a substantial advantage through random drift, which is insensitive to the form of epistasis.

\section{MAXIMUM RATE OF ADAPTATION}

The effects of random drift on the evolution of recombination are harder to analyze or to simulate than the effect of epistasis - the problem is stochastic, and strong effects emerge only when very many loci are involved. So, I concentrate here on a basic question that can be answered in a fairly simple way: How fast, in principle, can a population adapt? Of course, this question is interesting in itself: Just how effective, in principle, can selection be?

Suppose that beneficial mutations arise within a very large population, at a rate $U$ per genome per generation. Mutations act independently (i.e., there is no epistasis), and each of them multiplies fitness by a factor $(1+s)$. It is convenient to think of a trait, the log fitness, $z$, with each mutation adding to the trait, and fitness being $\exp (z)$. Then, if $z$ is normally distributed with variance $v$, the variance in fitness will be $V=e^{v}$. (Note that the trait $z$ is the genotypic value for log fitness; noninherited fitness variance can be included in the distribution of offspring number, conditioned on $z$.)

If we could ignore linkage, the chance that any allele will fix is just $2 s$, so there would be a baseline rate of substitution, in the absence of Hill-Robertson interference, of $\Lambda_{0}=2 s N U$. However, selection across the genome reduces the chance to $P<2 s$ that any one mutation will fix. This probability of fixation will be a decreasing function of the rate of sweeps occurring at other loci. So, $\Lambda=\Lambda_{0} P(\Lambda) / 2 s$. Thus, we can work out the net rate of sweeps if we know by how much that rate will reduce the chance of fixation, $P(\Lambda)$, of any new mutation. This is a huge simplification, because we do not need to worry about how new alleles increase all the way to fixation. Instead, we just focus on whether they can increase from one copy up to a large enough number to avoid random loss, although this is still rare overall.

The fate of a single gene introduced into a very large population can be found by assuming that all of its offspring reproduce independently of one another. Then, it follows a "branching process" that can be analyzed even when the gene may find itself in a variety of locations or genetic backgrounds. I first lay out some general results 
for branching processes, showing how fixation probability is related to the classical concept of "reproductive value." I then summarize results for unlinked genes and for a linear genetic map.

First, consider a single gene in an unstructured population. Suppose that it has a probability $\Psi_{k}$ of having $k$ offspring. Then, the chance that a copy at time $t$ will ultimately be lost, $1-P_{t}$, is just the sum over the chance that it leaves $k$ offspring and that all $k$ of these are lost $\left(1-P_{t+1}\right)^{k}$ :

$$
1-P_{t}=\sum_{k} \Psi_{k}\left(1-P_{t+1}\right)^{k}
$$

If the allele has a small selective advantage $s$ and therefore a small chance of being fixed $\left(P \sim_{S}<<1\right)$, we can expand Equation 1 as a Taylor series:

$$
1-P_{t}=\sum_{k} \psi_{k}\left(1-k P_{t+1}+\frac{k(k-1)}{2} P_{t+1}^{2} \ldots\right) \text {. }
$$

If the average number of offspring is $E[k]=1+s$, then we have (approximating to continuous time for $s<<1$ )

$$
-\partial_{t} P=s P-\frac{V}{2} P^{2}+\mathrm{O}\left(s^{3}\right)
$$

where $V$ is the variance in fitness. This equation applies with selection that changes through time, but if it is constant, we simply have $P=2 s / V$ : Fixation probability is reduced in proportion to the variance in fitness of individual genes. Note that the effective population size (defined via the rate of drift of neutral alleles) is reduced in the same way and so we could also write the more familiar form $P=2 s\left(N_{e} / N\right)$ (Whitlock and Gomulkiewicz 2005).

This derivation extends to structured populations, in which genes can be in different states, labeled $i$. These might represent spatial locations or, for our purposes, different genetic backgrounds. We will see that the overall fixation probability, averaged over states, is reduced by the variance in reproductive value across states, in much the same way that random variation in fitness between genes reduces it, as just discussed. Making the same approximation as before, we find that

$$
P_{i, t}=(1+s) \tilde{P}_{i, t}-\frac{\tilde{P}_{i, t}^{2}}{2}+\mathrm{O}\left(s^{3}\right)
$$

where $\tilde{P}_{i, t=} \sum_{j} M_{i, j} P_{j, t+1}$ is the expected fixation probability of a gene in an offspring from a parent in state $i$. This is an average over the different states $j$ in which the gene might be found in the next generation; $M_{i, j}$ is the chance that an offspring from a parent in $i$ will be in $j$, which here represents recombination between genetic backgrounds. Because we assume that selection is weak $(s<<1)$, we can again take time to be approximately continuous. Then, Equation 3 extends to

$$
-\partial_{t} P_{i}=s P_{i}+\sum_{j} M_{i, j}^{*} P_{j}-\frac{\tilde{P}_{i}^{2}}{2}+\mathrm{O}\left(s^{3}\right),
$$

where $M^{*}=M-I$ is the rate of movement between states.

A key concept is the "reproductive value" of a gene in state $i, v_{i}$, which is defined as the expected number of copies that the gene will leave in the distant future, normalized so that $E\left[v_{i}\right]$. For a weakly selected allele $(s<<$ $M)$, the fixation probability is proportional to the reproductive value (i.e., $P_{i} \sim v_{i}$ ). This is because if genes move between states much faster than the rate of selection (e.g., if recombination is much faster than selection), the relative contribution of a gene in state $i$ to any state $j$ will be proportional to its reproductive value after a long enough time; the probability that it will be picked up by selection is therefore also proportional to the reproductive value $v_{i}$.

Finally, we need to know the absolute fixation probability, averaged over states. Averaging Equation 4 over states leads to

$$
-\partial_{t} \bar{P}=s \bar{P}-\frac{E\left[P^{2}\right]}{2} .
$$

Because $E\left[P^{2}\right]>\bar{P}^{2}$, we see that variation in fixation probability across states necessarily reduces the average fixation probability. If the population is at equilibrium, we can write $E\left[P^{2}\right]>\bar{P}^{2}(1+C V)$, where $C V$ is the coefficient of variation of $P$ across states, and so $\bar{P}=2 s /(1+C V)$. This is consistent with Maruyama's (1971) invariance principle that the probability of fixation of an advantageous allele is equal to $2 s$ even in a structured population, provided gene flow does not alter allele frequencies. In that case, $P_{i}=2 s$ for all locations $i$ and is therefore not reduced below the panmictic value of $2 s$.

If mixing across states is rapid, then $P$ is proportional to reproductive value, and we immediately find that $\bar{P}=$ $2 s / E\left[v^{2}\right]$ - a remarkably simple result. We see that fixation probability, and hence the rate of adaptation, is reduced by a factor $1 / E\left[v^{2}\right]$. Therefore, recombination gives an advantage by shuffling genes across backgrounds and so reducing the variance in reproductive value $v$ between backgrounds. This is consistent with Hill and Robertson's (1966) account, in which selected loci interfere with one another by, in effect, inflating the rate of random drift. We can now apply these ideas to two specific models: a large number of unlinked loci or a linear genetic map.

\section{THE INFINITESIMAL MODEL}

In the very simplest case, suppose that fitness variation is not inherited. Uncorrelated variance in fitness $V$ will nevertheless reduce the fixation probability by a factor $1 / V=e^{-v}$. If the trait is heritable, being determined by a very large number of unlinked loci, fluctuations in fitness will be correlated across generations and so will cause much more random drift. Robertson (1961) argued that a gene in a background with value $\delta z$ above the trait mean will in the next generation find itself in a background with average value $\delta z / 2$. Thus, the excess, summed over generations, is expected to be $\delta z\left(1+\frac{1}{2}+\frac{1}{4} \ldots\right)=2 \delta z$. Therefore, the cumulative variance in the trait value will be multiplied by $2^{2}=4$. This heuristic argument suggests that heritable variance in log fitness will reduce fixation probability by a factor $e^{-4 v}$, rather than $e^{-v}$. This is confirmed by solving Equation 5. The reproductive value of 
a gene in a background with value $z$ is $e^{2 z-v}$, which is proportional to the square of the fitness and has expectation 1 . The mean square reproductive value is $e^{4 v}$, and so fixation probability is indeed reduced by a factor $e^{-4 v}$. If the rate of beneficial mutation is extremely high, all of the genetic variance is due to selective sweeps, and in a haploid population, $v=\Lambda \bar{s}$, where $\bar{s}=E\left[s^{2}\right] / E[s]$ is the average selection. Solving $\Lambda=\Lambda_{0} e^{-4 v}$, we find that the rate of adaptive substitutions only increases logarithmically with the input of favorable mutations (Fig. 3, middle line). This argument assumes that every offspring comes from randomly chosen parents; if instead parents mate for life, the effect of inherited fitness variation is much greater, because it is correlated across siblings. The reduction in fixation probability is then $e^{-9 v}$, rather than $e^{-4 v}$ (Fig. 3, lower line).

\section{UNLINKED LOCI: SEXUAL VERSUS ASEXUAL REPRODUCTION}

We cannot use the infinitesimal model to follow the evolution of recombination because all loci are unlinked, and so all recombination rates are fixed at $1 / 2$. However, we can model facultative sex by assuming that a fraction $\alpha$ reproduce asexually and produce identical offspring.

In an infinite population, under purely directional selection, both sexual and asexual reproduction lead to the same genetic variance and hence the same rate of adaptation. However, the reproductive value depends on the trait in a very different way for sexuals and asexuals: With asexual reproduction, the current trait value stays tied to the genotype indefinitely, and so reproductive value depends extremely steeply on the current trait value. We have seen that in a sexual population, with variance in $\log$ fitness $v$, the mean increases by $v$ in each generation, and the reproductive value in any generation is proportional to $e^{2 z}$. In contrast, the reproductive value of an asexual diverges and increases more and more steeply with $z$ : The number of offspring from an individual with trait value $z$ is proportional to $e^{z}$ after one generation, $e^{2 z}$ after two generations, and $e^{t z}$ after $t$ generations. However, the absolute number of offspring will always fall behind the number of sexuals, because the latter are steadily increasing their mean and must eventually overtake any given asexual lineage. In generation $t$, an asexual geno-

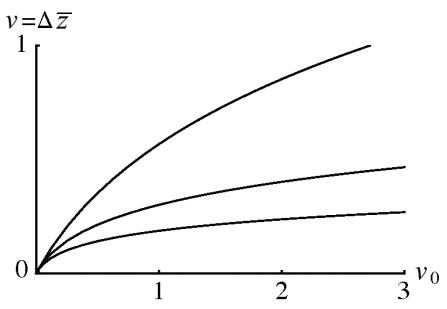

Figure 3. The rate of increase of $\log$ mean fitness $\Delta \bar{z}=v$, plotted against the baseline rate $V_{0}=2 N U s^{2}$, expected in the absence of interference between sweeps. (Top) Uncorrelated fitness fluctuations, (middle) unlinked loci, polygamy, (bottom) unlinked loci, monogamy. type $z$ has absolute fitness $e^{z-v t}$, and multiplying across generations, the number of asexuals is $\prod_{r=1}^{t} e^{z-v t}=\exp (z t$ $-v t[t-1] / 2)$. This has a maximum at $\exp \left([z+v / 2]^{2} /[2 v]\right)$, which is rather more than $\exp \left(k^{2} / 2\right)$ if $z=k \sqrt{v}$ standard deviations. Thus, an asexual lineage can increase to very large numbers in the short term (up to $t \sim[z / v]$ generations), but it must eventually fall behind a sexual population that can continually replenish its genetic variance by recombination (Fig. 4).

This contrast between selection on sexual and asexual populations was understood a century ago in the debate regarding the effectiveness of selection on Mendelian variation. Johanssen promoted the view that selection could only pick out the best genotype available in the $\mathrm{F}_{2}$ population, which would be the case if subsequent reproduction were asexual (Provine 1971). However, Castle's experiments, and many since, have shown that artificial selection can change the mean by many tens of standard deviations, using only standing variation (Barton and Keightley 2002).

Surprisingly, the same divergence of reproductive value is seen even if there is only a small rate $\alpha$ of asexual reproduction. Then, the reproductive value increases more steeply than $e^{2 z}$, and for sufficiently large $z$, it diverges over time; this is because the number of offspring produced entirely by asexual reproduction keeps increasing if $\alpha e^{z}>1$, so that their very high intrinsic fitness $e^{z}$ outweighs the loss due to mating back to the sexual population. This divergence implies that in a truly infinite population, the mean square reproductive value would diverge, and the fixation probability would tend to zero, even if individuals reproduced asexually only a small fraction of the time (Fig. 5).

In fact, even in a very large population, extremely fit individuals, many standard deviations above the mean, do not exist. Thus, deterministic arguments that require their existence will fail even in very large populations. (I ignore, for simplicity, the additional biological constraint on the maximum number of offspring.) Simulations suggest that indeed, a moderate rate of asexual reproduction slows the response to selection appreciably, because very fit lineages are lost by chance from the leading edge, causing a reduced variance (i.e., negative linkage disequilibria). Moreover, an unlinked modifier allele that causes a low rate of asexual reproduction can have a substantial

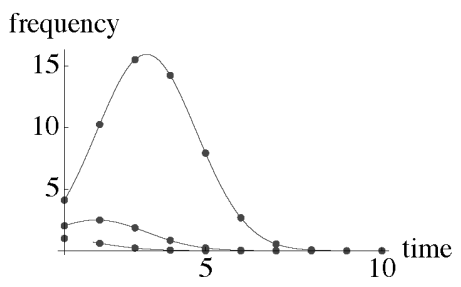

Figure 4. Frequency of an asexual clone that initially has trait value $z=0,1$, and 2 standard deviations above the mean of a sexual population, with variance in $\log$ fitness $v=0.5$. Because the $\log$ fitness of the sexual population grows as $z=v t$, the asexual population must eventually become extinct. (Generations are discrete; the lines connecting the dots are only for clarity.) 


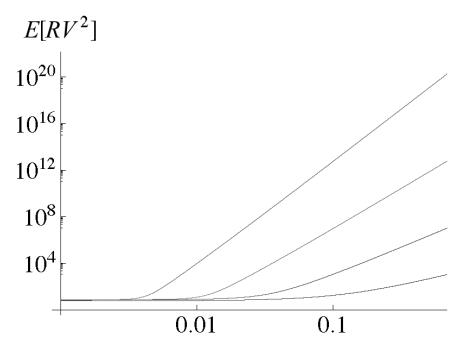

Figure 5. Mean square reproductive value, plotted against the probability of reproducing asexually $\alpha$, assuming the infinitesimal model and an infinite population. Values are plotted at $t=4$, $6,8,10$ generations (bottom to top). The variance in reproductive value diverges over time, because very fit individuals contribute an extremely large number of offspring.

disadvantage, even in large populations (Fig. 6). This is surprising, because selection on such a modifier is expected to be weak when it only stays associated with its effects for a short time (Barton 1995a).

This analysis of the infinitesimal model has depended only on standing variation and has not involved mutation, although the heritable variation in fitness must ultimately be sustained by mutation and might be due to the immediate effect of deleterious or beneficial mutations. All that matters in these models of loosely linked loci is the current standing variation. However, because the variance in reproductive value diverges, the fixation probability of new favorable mutations will be strongly reduced by selection, even if the rate of asexual reproduction is low.

\section{A LINEAR GENETIC MAP}

By how much do random mutations, scattered over a linear genetic map, reduce fixation probability? For any particular model of selection, this can be answered using Equation 4; now, the states $i$ correspond to the possible genetic backgrounds. For example, Figure 7 shows how the fixation probability depends on when and where on the map a new favorable mutation occurs, given that four sweeps are in progress at linked loci. Its chances are reduced below the baseline level of $2 s$ if it occurs just before a sweep or is closely linked to it. However, such calculations are not feasible for more than a few loci, because the number of different genetic backgrounds

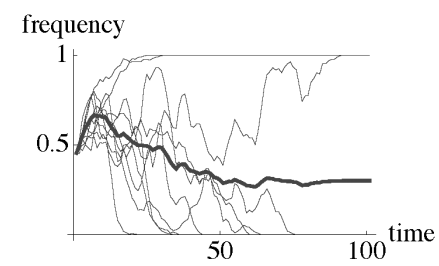

Figure 6. Frequency of a modifier that increases the rate of asexual reproduction from zero to $\alpha=20 \%$. Ten replicate simulations are shown of a population of 1000 haploid individuals. Although individual outcomes are highly variable, the average decline in frequency indicates a selective disadvantage of $-3.0 \%$ (thick line). Selection acts according to the infinitesimal model, with variance in $\log$ fitness $v=1$.

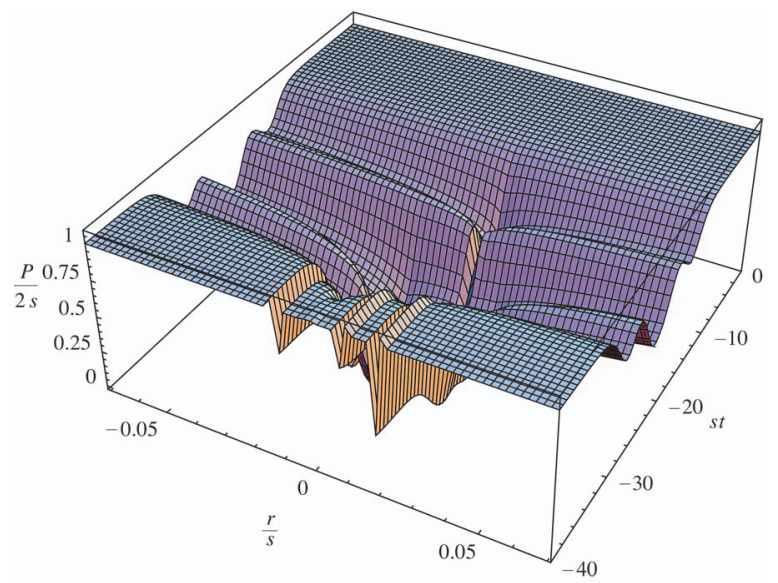

Figure 7. Fixation probability is reduced by four selective sweeps. The fixation probability $\frac{P}{2 s}$, measured relative to its baseline value $2 s$, is plotted against the map position of the new mutation $(r / s)$ and its time of occurrence $(s t)$. The four sweeps occur at evenly spaced map positions $r=0.01 \mathrm{~s}$ apart; mutations were introduced at times $s t=-5,-10,0,-5$, as single copies in a population of $N=10^{6}$.

increases so quickly. Moreover, we would really like to know the loss of fixation probability (i.e., the volume below the flat surface in Fig. 7), averaged over randomly scattered sweeps.

We can, however, make a scaling argument that shows how average fixation probability depends on selection strength, population size, and map length $(s, N, R)$. The key assumption is that $N$ is so large that the establishment of a new mutation is influenced by sweeps that are already established at high frequency and, if it survives, will itself influence mutations only far into the future. Thus, population size does not by itself directly affect the results. ( $N$ must be large enough that negative linkage disequilibria generated while it is rare have dissipated, but this effect is weak.) We can also assume that the map is so long that loosely linked genes have little effect; then, what matters is the density of substitutions per generation and per map length $\Lambda / R$. (Large numbers of completely unlinked genes, on other chromosomes, can be analyzed by the infinitesimal model, as above.) Finally, the strength of selection has two opposing effects: The length of map affected is approximately the strength of selection on the sweep $S$, whereas a new rare allele, with advantage $s$, is vulnerable for $\sim 1 / s$ generations. Averaging over a uniform density of times and positions on the map, these two effects cancel: What matters is the ratio $S / s$ between selection on the established sweep and on the new mutation. Overall, we find that density of sweeps $\Lambda / R$ is a decreasing function $f\left(\Lambda_{0} / R\right)$ of that rate. The dimensionless function $f$ depends on the shape of the distribution of selection but not on its absolute strength.

To obtain a concrete form for this function, we must make an approximation. If the established sweeps are much more strongly selected than the alleles that they chase out of the population, $S>>$ s, the fixation probability is closely approximated by $P=2\left(s-s^{*}\right)$, where $s^{*}$ is a critical selection coefficient proportional to the additive 
genetic variance per map length (Barton 1994). In a large population, subject to recurrent sweeps, a weakly selected allele has a negligible chance of fixation if its advantage is less than this critical value (more precisely, $P$ tends to zero as $N$ tends to infinity). This critical value is easily understood: It just counterbalances the expected rate of decrease of a new allele, due to sweeps that will almost always occur on another background (Fig. 8).

Using this approximation, we find that if all mutations have the same effect $(\Lambda / R) \sim\left(\Lambda_{0} / R\right) /\left(1+1.94\left[\Lambda_{0} / R\right]\right)$. This approximation works well for moderate rates of substitution but somewhat overestimates the degree of Hill-Robertson interference for high rates. Crucially, however, the scaling argument matches simulations well: Values converge as population size increases to a value that is independent of $s$ and $R$ (Fig. 9).

These arguments show that the maximum rate at which favorable mutations can be accumulated by selection is limited by recombination, via the Hill-Robertson effect. It is unlikely that most hitchhiking is due to selective sweeps, as was assumed here; deleterious mutations must also contribute. Hudson and Kaplan (1995) and Nordborg et al. (1996) showed how such "background selection" reduces neutral diversity, and Barton (1995b) showed that the effect on fixation probability is similar: For deleterious mutations scattered evenly over a linear genetic map, both are reduced by $\sim \exp (-U / R)$, independent of the strength of selection. This dimensionless ratio suggests that a similar scaling argument to that given above could be used to find the joint effects of many genes. (However, this result is essentially an extrapolation from the twolocus theory and, for multiple loci, is only an approximation; Barton 1995b.) Surprisingly, however, analysis of a population at mutation-selection balance is harder than that for multiple overlapping sweeps. This is because we must consider linkage disequilibria among common genotypes that typically carry many deleterious mutations. The analysis of multiple sweeps given above was greatly simplified, because under multiplicative selection in a large population, new alleles sweep through at close to linkage equilibrium.

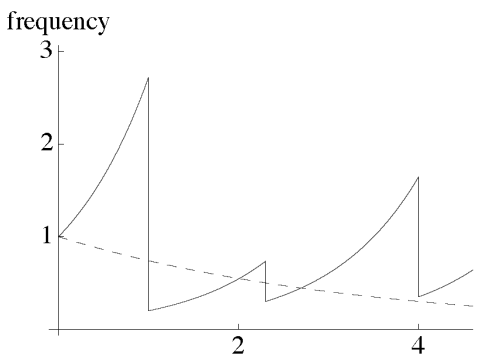

Figure 8. A rare allele will experience selective sweeps at linked loci as a series of random catastrophes that suddenly knock it back to a fraction of its previous frequency. If the rate of sweeps is above a threshold, a rare allele will on average decline and can never fix (dashed line). (Actually, there is a very small chance that the sweep will occur on coupling with the rare allele, giving it a very large boost. However, this can be neglected in large populations.)

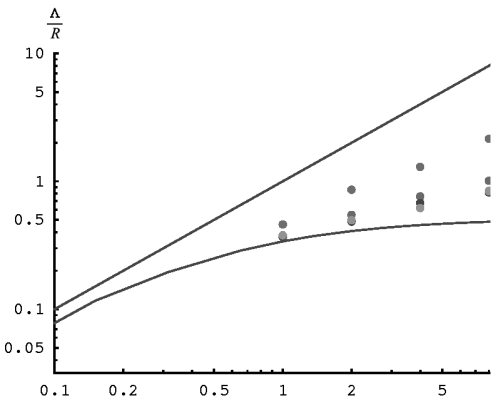

Figure 9. Rate of sweeps per unit map length, plotted against the baseline rate $\Lambda_{0} / R=2 s N U / R$. The series of dots are for population size $N=10^{2}, 10^{3}, \ldots 10^{5}$ (moving down). (Upper line) $\Lambda=$ $\Lambda_{0}$, (lower curve) the approximation $\left(\Lambda_{0} / R\right) /\left(1+1.94 Z\left[\Lambda_{0} / r\right]\right) . s$ $=0.05, R=1$; values are the mean of 10 replicates.

\section{DISCUSSION}

The most plausible explanation for the prevalence of sex and recombination is that it facilitates natural selection by maintaining high additive genetic variance in fitness. This requires that there are typically negative associations among favorable alleles, which hinder selection and must be broken up by recombination. Several simulation studies have shown that substantial negative associations can be generated by random drift, even in large populations (Otto and Barton 2001; Iles et al. 2003; Keightley and Otto 2006). This can be understood by realizing that even in a fairly large population, rare alleles - and in particular, new favorable mutationsare vulnerable to random loss, and their chances of fixation can be greatly increased by recombination, which frees them from random, and typically negative, association with their genetic background. Simple arguments, based on branching processes, show how this Hill-Robertson effect depends on the heritable variance in fitness $V$, on the density of deleterious mutations on the genetic map $U / R$, and on the density of selective sweeps $\Lambda / R$.

Although not discussed in detail here, this analysis extends to show how modifiers that increase recombination can gain an advantage if they are linked to adaptive variation. This can be done both for alleles that are at appreciable frequency, with random drift being treated as a perturbation (Barton and Otto 2005), and for new mutations that follow a branching process, as discussed above (Roze and Barton 2006). In both cases, although the analysis of two or three loci is fairly straightforward, extending results to large numbers of selected genes remains an important open question: It is just this realistic case that simulations suggest will most readily select for recombination (Iles et al. 2003; Keightley and Otto 2006).

How important is Hill-Robertson interference in nature? For unlinked loci, the key parameter is the heritable variance in fitness - the best measure of the total amount of selection that acts. In principle, the genetic variance of fitness could be estimated from the number of offspring in successive generations, requiring simply a large pedigree. Unfortunately, it has been extremely difficult to measure this fundamental quantity in any organism. Large, long-term surveys are needed to estimate the 
genetic variance of any quantitative trait, and fitness is especially problematic because it involves the whole life cycle and is perturbed by noninherited variation (Houle 1992). Thus, although the heritability of fitness (the ratio of the genetic variance to the total) is low, the heritable variance itself may well be high - high enough to reduce the rate of adaptation by more than an order of magnitude. (For example, if $V=e^{v}=2$, fixation probability would be reduced by a factor $e^{-4 v}=V^{4}=0.0625$ in a polygamous species and by $e^{-9 v}=V^{-9}=0.002$ in a monogamous species.) (For reviews, see Burt [2000]; Merila and Sheldon [2000].)

Recently, it has become feasible to measure the total mutation rate directly by comparing DNA sequences across several generations (Haag-Liautard et al. 2007; Lynch et al. 2008) - a great improvement on previous indirect techniques (Drake et al. 1998). It is also possible to estimate the fraction of sequence that is constrained by selection, by comparing rates of molecular evolution with presumed neutral sequence, such as pseudogenes. These recent estimates suggest that the rate of deleterious mutation may be large ( $U$ $>1$, say) in organisms with large functional genomes, such as ourselves. Thus, deleterious mutation may be maintaining high recombination rates in such organisms and clearly does interfere with selection in genomic regions of low recombination (e.g., in Y chromosomes; Charlesworth et al., this volume). However, it does not seem to provide an explanation for high rates of recombination in eukaryotes in general, for which $U$ may be much lower (Lynch et al.2008).

We now have good estimates of the rate of divergence between species and the fraction that is due to positive selection (see, e.g., Smith and Eyre-Walker 2002). Although a large number of selected substitutions distinguish sister species, the rate of species-wide sweeps per generation must be very low-much too low to cause significant selection for recombination (Roze and Barton 2006). We know that episodes of intense selection can select for higher recombination-recombination rates increase significantly following artificial selection (for review, see Otto and Barton 2001). It may be that such selection is widespread in nature: Local selective sweeps could be frequent enough to maintain high rates of recombination (Martin et al. 2006; Roze 2009) and yet need not contribute significantly to the rate of species-wide sweeps. Genome-wide surveys of geographic differentiation may soon settle this question. For example, Pickrell et al. (2009) use human single-nucleotide polymorphism (SNP) data from 53 populations to identify several examples of loci that have undergone recent positive selection in limited geographic areas; however, Coop et al. (2009) note that there are surprisingly few examples where different alleles are fixed or nearly fixed in diferent human populations. Although the pattern is as yet unclear, such data do have the potential to tell us the rate at which alleles sweep through local populations.

\section{ACKNOWLEDGMENTS}

I thank the Royal Society and the Engineering and Physical Sciences for support (GR/ T11753/01). Jonathan Coe provided the simulations for Figure 6.

\section{REFERENCES}

Barton NH. 1994. The reduction in fixation probability caused by substitutions at linked loci. Genet Res 64: 199-208.

Barton NH. 1995a. A general model for the evolution of recombination. Genet Res 65: 123-144.

Barton NH. 1995b. Linkage and the limits to natural selection. Genetics 140: 821-841.

Barton NH, Keightley PD. 2002. Understanding quantitative genetic variation. Nat Rev Genet 3: 11-21.

Barton NH, Otto SP. 2005. Evolution of recombination due to random drift. Genetics 169: 2353-2370.

Bonhoeffer S, Chappey C, Parkin NT, Whitcomb JM, Petropoulos CJ. 2004. Evidence for positive epistasis in HIV-1. Science 306: $1547-1551$.

Burger R. 1999. Evolution of genetic variability and the advantage of sex and recombination in changing environments. Genetics 153: 1055-1069.

Burt A. 2000. Sex, recombination and the efficacy of selection-Was Weissman right? Evolution 54: 337-351.

Butlin R. 2002. The costs and benefits of sex: New insights from old asexual lineages. Nat Rev Genet 3: 311-317.

Charlesworth B. 1993. Directional selection and the evolution of sex and recombination. Genet Res 61: 205-224.

Charlesworth B, Barton NH. 1996. Recombination load associated with selection for increased recombination. Genet Res 67: 27-41.

Coop G, Pickrell JK, Novembre J, Kudaravalli S, Li J, Absher D, Myers RM, Cavalli-Sforza LL, Feldman MW, Pritchard JK. 2009. The role of geography in human adaptation. PLoS Genet 5: e1000500.

Darwin C. 1871. The descent of man, and selection in relation to sex. Murray, London.

Darwin CR. 1876. The effects of cross and selffertilisation in the vegetable kingdom. Murray, London.

de Visser JA, Elena SF. 2007. The evolution of sex: Empirical insights into the roles of epistasis and drift. Nat Rev Genet $\mathbf{8}$ : 139-149.

Drake JW, Charlesworth B, Charlesworth D, Crow JF. 1998. Rates of spontaneous mutation. Genetics 148: 1667-1686.

Edwards AWF. 2002. The fundamental theorem of natural selection. Theor Popul Biol 61: 335-338.

Elena SF, Lenski RE. 1997. Test of synergistic interactions among deleterious mutations in bacteria. Nature 390: 395-298.

Elena SF, Lenski RE. 2001. Epistasis between new mutations and genetic background and a test of genetic canalization. Evolution 55: 1746-1752.

Fisher RA. 1930. The genetical theory of natural selection. Oxford University Press, Oxford.

Haag-Liautard C, Dorris M, Maside X, Macaskill S, Halligan DL, Houle D, Charlesworth B, Keightley PD. 2007. Direct estimation of per nucleotide and genomic deleterious mutation rates in Drosophila. Nature 445: 82-85.

Hill WG, Robertson A. 1966. The effect of linkage on limits to artificial selection. Genet Res 8: 269-294.

Houle D. 1992. Comparing evolvability and variability of quantitative traits. Genetics 130: 195-204.

Hudson RR, Kaplan NL. 1995. Deleterious background selection with recombination. Genetics 141: 1605-1617.

Iles MM, Walters JR, Cannings C. 2003. Selection for recombination in large genomes. Genetics 165: 2249-2258.

Keightley PD, Otto SP. 2006. Interference among deleterious mutations favours sex and recombination in finite populations. Nature 443: 89-92.

Kirschner M, Gerhart J. 1998. Evolvability. Proc Natl Acad Sci 95: 8420-8427.

Kouyos RD, Otto SP, Bonhoeffer S. 2006. Effect of varying epistasis on the evolution of recombination. Genetics 173: 589-597.

Kouyos RD, Silander OK, Bonhoeffer S. 2007. Epistasis between deleterious mutations and the evolution of recombination. Trends Ecol Evol 22: 308-315.

Koza JR, Bennett FH, Andre D, Keane MA. 2002. Genetic programming: Biologically inspired computation that creatively solves non-trivial problems. In Evolution as computation (ed. 
LF Landweber and E Winfree), pp. 95-124. Springer, Berlin. Lynch M, Sung W, Morris K, Coffey N, Landry CR, Dopman EB, Dickinson WJ, Okamoto K, Kulkarni S, Hartl DL, Thomas WK. 2008. A genome-wide view of the spectrum of spontaneous mutations in yeast. Proc Natl Acad Sci 105: 9272-9277.

Martin G, Otto SP, Lenormand T. 2006. Selection for recombination in structured populations. Genetics 172: 593-609.

Maruyama T. 1971. An invariant property of a structured population. Genet Res 18: 81-84.

Maynard Smith J. 1978. The evolution of sex. Cambridge University Press, Cambridge.

Merila J, Sheldon B. 2000. Lifetime reproductive success and heritability in nature. Am Nat 155: 301-310.

Mitchell M. 1998. An introduction to genetic algorithms. MIT Press, Cambridge, MA.

Muller HJ. 1932. Some genetic aspects of sex. Am Nat 66: 118138.

Nordborg M, Charlesworth B, Charlesworth D. 1996. The effect of recombination on background selection. Genet Res 67: 159174

Otto SP, Barton NH. 2001. Selection for recombination in small populations. Evolution 55: 1921-1931.

Otto SP, Feldman MW. 1997. Deleterious mutations, variable epistatic interactions, and the evolution of recombination. Theor Popul Biol 51: 134-147.

Pickrell JK, Coop G, Novembre J, Kudaravalli S, Li JZ, Absher D, Srinivasan BS, Barsh GS, Myers RM, Feldman MW, Pritchard JK. 2009. Signals of recent positive selection in a worldwide sample of human populations. Genome Res 19: 826-837.
Price GR. 1972. Fisher's "fundamental theorem" made clear. Ann. Hum. Genet 36: 129-140.

Provine W. 1971. The origins of theoretical population genetics. University of Chicago Press, Chicago, IL.

Robertson A. 1961. Inbreeding in artificial selection programmes. Genet Res 2: 189-194.

Roze D. 2009. Diploidy, population structure and the evolution of recombination. Am Nat 174: S79-S94.

Roze D, Barton NH. 2006. The Hill-Robertson effect and the evolution of recombination. Genetics 173: 1793-1811.

Smith NGC, Eyre-Walker A. 2002. Adaptive protein evolution in Drosophila. Nature 415: 1022-1024.

Sniegowski PD, Murphy HA. 2006. Evolvability. Curr Biol 16: R831-R834.

Szathmary E. 1993. Do deletion mutations act synergistically? Metabolic control theory provides a partial answer. Genetics 133: $127-132$.

Wagner GP, Altenberg L. 1996. Complex adaptations and the evolution of evolvability. Evolution 50: 967-976.

Waxman D, Peck JR. 1999. Sex and adaptation in a changing environment. Genetics 153: 1041-1053.

Weismann A. 1889. The significance of sexual reproduction in the theory of natural selection. In Essays upon heredity and kindred biological problems (ed. EB Poulton et al.). Clarendon, Oxford.

Whitlock MC, Gomulkiewicz R. 2005. Probability of fixation in a heterogeneous environment. Genetics 171: 1407-1417.

Williams GC. 1975. Sex and evolution. Princeton University Press, Princeton, NJ. 


\section{$\$_{\mathrm{CSH}}^{\infty}$ Cold Spring Harbor Symposia SYMPOSIA on Quantitative Biology}

\section{Why Sex and Recombination?}

N.H. Barton

Cold Spring Harb Symp Quant Biol 2009 74: 187-195 originally published online November 10, 2009 Access the most recent version at doi:10.1101/sqb.2009.74.030

References This article cites 45 articles, 4 of which can be accessed free at: http://symposium.cshlp.org/content/74/187.full.html\#ref-list-1

License

Email Alerting Receive free email alerts when new articles cite this article - sign up in the box at the Service top right corner of the article or click here.

To subscribe to Cold Spring Harbor Symposia on Quantitative Biology go to: http://symposium.cshlp.org/subscriptions 